\title{
Blood pressure variability correlates with right ventricular strain in women with gestational hypertension and preeclampsia
}

\author{
Marijana Tadic $\mathbb{D i D}^{1 凶}$, Cesare Cuspidi ${ }^{2}$, Jelena Suzic Lazic ${ }^{1}$, Vladan Vukomanovic ${ }^{1}$, Sladjana Mihajlovic ${ }^{3}$, Predrag Savic ${ }^{1}$, Marko Cvrkotic $^{1}$, \\ Guido Grassi ${ }^{4}$ and Vera Celic ${ }^{1}$
}

(c) The Author(s), under exclusive licence to Springer Nature Limited 2021

\begin{abstract}
The aim of this study was to evaluate the short- and long-term blood pressure (BP) variability and right ventricular (RV) remodeling in women with gestational hypertension and preeclampsia, as well as their association. This cross-sectional study included 161 pregnant women (56 normotensive controls, 55 patients with gestational hypertension, and 50 patients with preeclampsia) after 20 weeks of gestation. All women underwent 24-h ambulatory BP monitoring and echocardiographic examination. Our findings showed that 24-h, daytime and nighttime systolic and diastolic BPs, as well as visit-to-visit systolic and diastolic BPs, were significantly higher in women with gestational hypertension and preeclampsia than in control group. Parameters of short- and long-term BP variability gradually increased from controls, throughout women with preeclampsia, to those with gestational hypertension. RV diameter, E/e' and PAP were significantly higher in women with gestational hypertension and preeclampsia than in controls. Global and free wall RV longitudinal strains, as well as corresponding endo- and epicardial strains, gradually reduced from controls to women with preeclampsia. Parameters of short- and long-term BP variability were independently associated with global and free wall RV longitudinal strain. In conclusion, short- and long-term BP variability was higher in women with pregnancy-induced hypertensive disorders. RV diastolic function and mechanics were deteriorated in these women comparing with controls. A significant association between BP variability and RV longitudinal strain underlines the importance of determination of short- and long-term BP variability during pregnancy.
\end{abstract}

Journal of Human Hypertension (2022) 36:826-832; https://doi.org/10.1038/s41371-021-00580-x

\section{INTRODUCTION}

Hypertensive disorders in pregnancy represent one of the most common complications during gestation. This entity includes several hypertensive disorders (pregestational hypertension, gestational hypertension, preeclampsia, and preeclampsia superimposed on chronic hypertension) and all of them are associated with increased morbidity and mortality during pregnancy and particularly in its late stage [1]. Some studies even confirmed increased mortality risk, Alzheimer's disease, diabetes, ischemic heart disease, and stroke among women who experienced hypertensive disease of pregnancy [2]. The complexity of this type of hypertensive disorder is multisystem organ damage which involves heart, kidneys, liver, and vascular system.

Left ventricular (LV) remodeling in hypertensive disorders in pregnancy and particularly in gestational hypertension and preeclampsia has been extensively investigated in the last few decades [3-5]. However, right ventricular (RV) structural, functional, and mechanical changes are still mainly unrevealed. Scarce data are conflicting because some authors reported significant functional changes in terms of increased RV diameter, elevated pulmonary pressure and reduced RV longitudinal mechanicsstrain [6-9], whereas other studies demonstrated no difference in RV structure and function between normotensive controls and pregnant women with hypertensive disorders [10,11].
The effect of blood pressure (BP) variability in pregnancy is also uncertain. Data are scarce and mainly based on mid- and long-term $B P$ variability that was based on office BP measurements, which were made during each clinical visit-visit-to-visit variability [12, 13]. Studies showed that visit-to-visit BP variability was associated with gestational hypertension and preeclampsia [12], as well as with risk of adverse birth outcomes in pregnant women without proteinuria or chronic hypertension [13]. Recent investigation reported that short-term BP variability obtained by $24-\mathrm{h}$ ambulatory BP monitoring correlated with subclinical echocardiographic changes and microalbuminuria in normotensive women with history of preeclampsia or eclampsia [14]. The relationship between BP variability and RV remodeling has not been investigated so far.

The current study aimed to investigate RV structural, functional and mechanical changes, short- and long-term BP variability, as well as their relationship in women with gestational hypertension and preeclampsia.

\section{METHODOLOGY}

The present cross-sectional investigation involved 161 pregnant women (56 normotensive controls, 55 patients with gestational hypertension, and 50 patients with preeclampsia) after 20 weeks of gestation. Controls were selected from healthy pregnant women

\footnotetext{
'University Clinical Hospital Center "Dr. Dragisa Misovic-Dedinje", Clinic for Internal medicine, Cardiology Department, Belgrade, Serbia. ${ }^{2}$ University of Milan-Bicocca and Istituto,

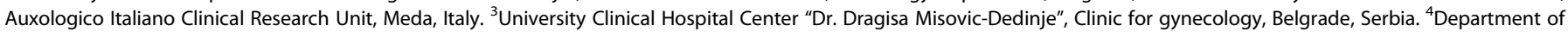

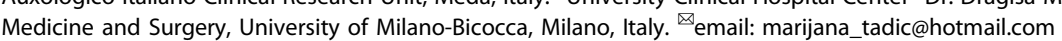


who were of similar age and gestational age as patients with gestational hypertension and preeclampsia. Gestational hypertension was defined as office systolic $B P \geq 140 \mathrm{mmHg}$ and diastolic $\mathrm{BP} \geq 90 \mathrm{mmHg}$ occurring after 20 weeks of gestation with no proteinuria [15]. Preeclampsia was defined as BP $\geq 140 \mathrm{mmHg}$ systolic or $\geq 90 \mathrm{mmHg}$ diastolic BP on 2 different occasions, and a urine protein $\geq 300 \mathrm{mg}$ in $24 \mathrm{~h}$ occurring after 20 weeks of gestation [15]. Patients with congenital heart disease, valvular heart disease, coronary artery disease, pulmonary disease, diabetes mellitus excluding gestational diabetes, and inadequate echocardiographic images (3 women) were excluded from the study.

Anthropometric measures (height, weight) and laboratory analyses (fasting glucose level, total cholesterol, creatinine, hemoglobin level and 24-h urine protein level), as well as current antihypertensive treatment, were investigated in all patients. Body mass index (BMI) and body surface area (BSA) were calculated for all patients. All women underwent a 24-h BP monitoring and echocardiographic examination. The echocardiographic examination was performed at the same day when 24-h ABPM was performed (just before putting BP monitor or just after removing it next day). The study was approved by the local Ethics Committee, and informed consent was obtained from all the participants.

\section{Clinic BP and 24-h ambulatory BP measurements}

Clinic arterial BP values were obtained by a calibrated sphygmomanometer in the morning hours by measuring the average value of the two consecutive measurements in the sitting position. BP was measured $\geq 2$ times in separate occasions before hypertension disorder was diagnosed and $\geq 5$ after hypertension was diagnosed. These values were used for calculation of visit-to-visit BP variability.

Schiller BR-102 plus system (Schiller AG, Baar, Switzerland) was used for noninvasive 24-h ambulatory BP monitoring. The device was programmed to obtain BP readings at 30-min intervals during the whole day. The recording was then analyzed to obtain a 24-h, daytime and nighttime average systolic and diastolic blood pressure according to the guidelines [16].

24-h BP variability has been evaluated by two different group of indices: (i) standard deviation (SD) of average 24-h BPs; and (ii) coefficient of variation (CV) of daytime, nighttime, and 24-h BPs that represents the average SD of BP divided by the corresponding mean BP and multiplied by $100[\mathrm{CV}=(\mathrm{SD} / \mathrm{BP}$ average values) $\times 100$ ]. Nocturnal $B P$ decline was calculated for all patients.

The visit-to-visit variability was determined by SD or CV of either systolic or diastolic BP from baseline through delivery.

\section{Echocardiography}

Echocardiographic examination was performed by a Vivid 95 ultrasound machine (GE Healthcare, Horten, Norway). The values of all 2D parameters were obtained as the average value of three consecutive cardiac cycles. LV diameters, interventricular septum, posterior wall thickness and relative wall thickness were evaluated in long-axis parasternal view [17]. LV ejection fraction (EF) was assessed by the modified Simpson's method of discs. LV mass was calculated by using the formula of the American Society of Echocardiography [17], and indexed for BSA.

Transmitral Doppler inflow and tissue Doppler velocities were obtained in the apical 4-chamber view. Pulsed Doppler measurements included the ratio between the transmitral early and late diastolic peak flow velocity (E/A). Tissue Doppler imaging was used to obtain LV myocardial velocities at the septal and lateral segment of the mitral annulus during early and late diastole ( $\mathrm{e}^{\prime}$ and $\left.\mathrm{a}^{\prime}\right)$.

\section{Right ventricle and atrium}

The RV internal diameter was measured in the basal RV segment in apical 4-chamber view [18]. RV thickness was measured in the subcostal view. RA maximal volume was obtained in the 4-chamber view during ventricular end-systole and indexed for
BSA. Fractional area change (FAC) was calculated as the percentage of change in RV area during systole: (end-diastolic area - end-systolic area)/end-diastolic area [18]. Tricuspid inflow (E) and tissue Doppler velocities $\left(\mathrm{e}_{\mathrm{t}}^{\prime}, \mathrm{s}_{\mathrm{t}}\right)$ were evaluated in the apical 4-chamber view [18], and $E / e^{\prime}{ }_{t}$ ratio was calculated. RV systolic blood pressure (PAP) was assessed in the patients with minimal/mild tricuspid regurgitation and it was feasible to calculate in $144(89 \%)$ of patients. Tricuspid annular plane systolic excursion (TAPSE) has been measured in all participants, according to the guidelines [18].

\section{Two-dimensional right ventricular strain and strain rate} 2D strain imaging was performed by using 3 consecutive cardiac cycles of 2DE images in the apical 4-chamber view. EchoPAC 202 (GE-Healthcare, Horten, Norway), as a commercially available software, was used for the 2D strain analysis.

The automatic tracking of the endocardial contour was performed in end-systole and it was carefully verified, the region of interest was manually corrected to ensure optimal tracking and inclusion of the entire RV thickness. After delineating the region of interest, software allowed the investigation of 3 myocardial layers: endocardial, mid-myocardial and epicardial. Mid-myocardial strain is calculated as the average of endo- and epicardial strain and it was equal to global longitude al strain. All strain parameters were determined for the RV lateral wall and global RV, separately.

\section{Statistical analysis}

Continuous variables were presented as mean \pm SD and were compared by the analysis of equal variance (ANOVA), as they showed normal distribution. Tukey HSD post hoc analysis was used for the comparison between different groups. Differences in proportions were compared by the $x^{2}$ test. Univariable and multivariable regression analyses were used for determining the association between different BP variability and echocardiographic parameters. Four models for multivariable regression analyses were used to determine the association between BP variability indices and RV global longitudinal and free wall strain.

Model 1 consists of age, BMl, use of antihypertensive medications, LVEF, LVMI, E/e', 24-h SBP, and SD (24-h SBP)). Model 2 involves age, BMI, use of antihypertensive medications, LVEF, LVMI, E/e', 24-h SBP, and CV (24-h SBP). Model 3 includes age, BMI, use of antihypertensive medications, LVEF, LVMI, E/e', Vto-V SBP, and V-to-V SD (24-h SBP). Model 4 involves age, BMI, use of antihypertensive medications, LVEF, LVMI, E/e', V-to-V SBP, V-toV CV (24-h SBP), and V-to-V ARV (SBP). The $p$ value $<0.05$ was considered statistically significant.

\section{RESULTS}

There was no difference in age between controls, women with gestational hypertension and preeclampsia (Table 1). BMI was higher in patients with preeclampsia than in controls (Table 1). Laboratory parameters (plasma glucose, serum creatinine levels, cholesterol, and hemoglobin level) were similar between three groups (Table 1). 24-h proteinuria gradually increased from control group, across women with gestational hypertension, to those with preeclampsia (Table 1). There was no difference in prevalence of gestational diabetes between three observed groups and the use of antihypertensive therapy between patients with gestational hypertension and preeclampsia (Table 1). Gestational week at the time of examination (24-h ABPM and echocardiographic evaluation) was similar between three groups (Table 1).

\section{Ambulatory blood pressure monitoring and clinical BP measurements}

24-h, daytime and nighttime systolic and diastolic BP were higher in women with gestational hypertension and preeclampsia than in controls (Table 2). BP variability parameters-SD and CV gradually 
Table 1. Demographic characteristics and clinical parameters of study population.

\begin{tabular}{|c|c|c|c|c|}
\hline & Controls $(n=56)$ & Gestational hypertension $(n=55)$ & Preeclampsia $(n=50)$ & $p$ \\
\hline Age (years) & $30 \pm 4$ & $31 \pm 5$ & $31 \pm 5$ & 0.436 \\
\hline BMI $\left(\mathrm{kg} / \mathrm{m}^{2}\right)$ & $28.2 \pm 5.4$ & $29.0 \pm 6.1$ & $31.1 \pm 5.9^{\mathrm{a}}$ & 0.033 \\
\hline Parity & $1(1-3)$ & $2(1-3)$ & $2(1-3)$ & 0.837 \\
\hline Gestational age (weeks) & $31 \pm 4$ & $31 \pm 3$ & $32 \pm 4$ & 0.285 \\
\hline Plasma glucose $(\mathrm{mmol} / \mathrm{l})$ & $4.9 \pm 0.4$ & $4.8 \pm 0.5$ & $5.0 \pm 0.6$ & 0.129 \\
\hline Total cholesterol (mmol/l) & $4.6 \pm 0.5$ & $4.7 \pm 0.6$ & $4.7 \pm 0.6$ & 0.568 \\
\hline 24-h urine protein level (mg/day) & $86 \pm 21$ & $143 \pm 35$ & $969 \pm 311^{a, b}$ & $<0.001$ \\
\hline Gestational diabetes (\%) & $1(1.5)$ & $2(3.6)$ & $2(4)$ & 0.774 \\
\hline Antihypertensive therapy (\%) & - & $22(40)$ & $24(48)$ & 0.674 \\
\hline Gestational age at time of examination (weeks) & $32 \pm 3$ & $31 \pm 4$ & $32 \pm 4$ & 0.266 \\
\hline
\end{tabular}

$B M I$ body mass index.

${ }^{a} p<0.05$ for controls vs. preeclampsia.

${ }^{\mathrm{b}} p<0.01$ for preeclampsia vs. gestational hypertension.

Table 2. Ambulatory blood pressure measurements.

Controls $(n=56) \quad$ Gestational hypertension $(n=55) \quad$ Preeclampsia $(n=50) \quad p$

24-h ambulatory blood pressure monitoring

24-h

\begin{tabular}{|c|c|c|c|c|}
\hline SBP $(\mathrm{mmHg})$ & $108 \pm 6$ & $123 \pm 7^{\mathrm{a}}$ & $121 \pm 8^{b}$ & $<0.001$ \\
\hline $\mathrm{DBP}(\mathrm{mmHg})$ & $67 \pm 4$ & $76 \pm 5^{a}$ & $74 \pm 4^{b}$ & $<0.001$ \\
\hline \multicolumn{5}{|l|}{ aytime } \\
\hline $\mathrm{SBP}(\mathrm{mmHg})$ & $113 \pm 7$ & $128 \pm 9$ & $125 \pm 8$ & $<0.001$ \\
\hline DBP $(\mathrm{mmHg})$ & $70 \pm 4$ & $79 \pm 5^{\mathrm{a}}$ & $77 \pm 6^{b}$ & $<0.001$ \\
\hline \multicolumn{5}{|l|}{ ghttime } \\
\hline $\mathrm{SBP}(\mathrm{mmHg})$ & $100 \pm 5$ & $111 \pm 6^{a}$ & $110 \pm 7^{b}$ & $<0.001$ \\
\hline $\mathrm{DBP}(\mathrm{mmHg})$ & $61 \pm 4$ & $70 \pm 5^{a}$ & $68 \pm 5^{c}$ & $<0.001$ \\
\hline
\end{tabular}

Nocturnal reduction rate (\%)

$\begin{array}{lllll}\text { SBP (\%) } & 11.7 \pm 3.1 & 13.1 \pm 3.8 & 12.2 \pm 3.4 & 0.100 \\ \text { DBP (\%) } & 12.7 \pm 3.4 & 11.5 \pm 2.9 & 11.6 \pm 3.0 & 0.084\end{array}$

SD

CV

$24 \mathrm{~h} \mathrm{SBP} \quad 8.0 \pm 1.5$

$14.1 \pm 2.5$

$12.1 \pm 2.3$

$<0.001^{* *}$

Clinic blood pressure measurement

$\begin{array}{lllll}\text { SBP }(\mathrm{mmHg}) & 116 \pm 6 & 133 \pm 9^{\mathrm{a}} & 130 \pm 7^{\mathrm{b}} & <0.001 \\ \text { DBP }(\mathrm{mmHg}) & 72 \pm 4 & 83 \pm 6 & 80 \pm 6 & <0.001^{*} \\ \text { SD } & 8.0 \pm 1.9 & 15.3 \pm 3.3 & 12.8 \pm 3.1 & <0.001^{* *} \\ \text { CV } & 7.6 \pm 1.3 & 13.1 \pm 2.3 & 11.0 \pm 2.0< & <0.001^{* *}\end{array}$

$D B P$ diastolic blood pressure, $C V$ coefficient of variation, $S B P$ systolic blood pressure, $S D$ standard deviation.

${ }^{*} p<0.05$ for all comparisons, ${ }^{* *} p<0.01$ for all comparisons.

${ }^{a} p<0.01$ for controls vs. gestational hypertension.

$\mathrm{b}_{p}<0.01$ for controls vs. preeclampsia.

$c_{p}<0.01$ for gestational hypertension vs. preeclampsia.

increased from controls, across women with preeclampsia, to those with gestational hypertension.

Visit-to-visit systolic and diastolic BPs were higher in patients with gestational hypertension and preeclampsia than in controls (Table 2). Visit-to-visit BP variability (SD and CV) gradually increased from controls, across preeclampsia, to gestational hypertension.

\section{Echocardiographic parameters}

LV diameters, septum and relative wall thickness, as well as LVMI, were higher in patients with gestational hypertension and preeclampsia than in controls (Table 3). LVEF was similar across three groups. Patients with gestational hypertension and preeclampsia had significantly lower E/A ratio than controls. Mitral E/e 
Table 3. Echocardiographic parameters of left ventricular structure and function in the study population.

\begin{tabular}{|c|c|c|c|c|}
\hline & Controls $(n=56)$ & Gestational hypertension $(n=55)$ & Preeclampsia $(n=50)$ & $p$ \\
\hline \multicolumn{5}{|l|}{ LV parameters } \\
\hline LVEDD (mm) & $46 \pm 4$ & $47 \pm 4$ & $48 \pm 3^{b}$ & 0.024 \\
\hline $\operatorname{LVESD}(\mathrm{mm})$ & $26 \pm 3$ & $30 \pm 4^{\mathrm{a}}$ & $31 \pm 4^{b}$ & $<0.001$ \\
\hline IVS (mm) & $7.6 \pm 1.0$ & $8.2 \pm 1.1^{\mathrm{a}}$ & $8.4 \pm 1.2^{\mathrm{b}}$ & $<0.001$ \\
\hline RWT & $0.33 \pm 0.02$ & $0.35 \pm 0.04^{\mathrm{a}}$ & $0.35 \pm 0.03^{b}$ & $<0.001$ \\
\hline LVMI $\left(g / \mathrm{m}^{2}\right)$ & $63 \pm 7$ & $77 \pm 12^{\mathrm{a}}$ & $74 \pm 11^{\mathrm{b}}$ & $<0.001$ \\
\hline $\mathrm{E} / \mathrm{e}_{\mathrm{m}}^{\prime}$ ratio & $5.5 \pm 1.2$ & $6.8 \pm 1.4$ & $8.2 \pm 2.3$ & $<0.001^{* *}$ \\
\hline \multicolumn{5}{|l|}{ RV parameters } \\
\hline RV basal diameter (mm) & $23 \pm 2$ & $27 \pm 3^{\mathrm{a}}$ & $28 \pm 3^{b}$ & $<0.001$ \\
\hline RV thickness (mm) & $3 \pm 0.5$ & $3.2 \pm 0.8$ & $3.1 \pm 0.6$ & 0.268 \\
\hline $\mathrm{s}_{\mathrm{t}}(\mathrm{cm} / \mathrm{s})$ & $14 \pm 3$ & $13 \pm 3$ & $13 \pm 2$ & 0.089 \\
\hline PAP $(\mathrm{mmHg})$ & $23 \pm 3$ & $27 \pm 4$ & $30 \pm 4$ & $<0.001^{* *}$ \\
\hline \multicolumn{5}{|c|}{ Right ventricular strain parameters } \\
\hline \multicolumn{5}{|l|}{ Global longitudinal strain (\%) } \\
\hline Global RV & $-24.5 \pm 3.3$ & $-22.1 \pm 2.8$ & $-20.6 \pm 2.5$ & $<0.001^{*}$ \\
\hline Free wall RV strain (\%) & $-26.3 \pm 4.2$ & $-24.4 \pm 3.9$ & $-22.5 \pm 3.5$ & $<0.001^{*}$ \\
\hline \multicolumn{5}{|c|}{ Layer-specific longitudinal strain for global RV (\%) } \\
\hline Endocardial & $-26.1 \pm 3.9$ & $-24.3 \pm 3.2$ & $-22.1 \pm 3.0$ & $<0.001^{*}$ \\
\hline Epicardial & $-22.8 \pm 3.1$ & $-19.9 \pm 2.5^{\mathrm{a}}$ & $-19.0 \pm 2.1^{\mathrm{b}}$ & $<0.001$ \\
\hline
\end{tabular}

' gradually and significantly increased from control group to women with preeclampsia (Table 3).

RV diameter was significantly higher in women with gestational hypertension and preeclampsia than in controls (Table 3). RV thickness, TAPSE and $s^{\prime}$ were similar between observed groups. FAC was lower in women with gestational hypertension and preeclampsia than in controls. RAVI was higher in women with women with pregnancy-induced hypertension than in control group (Table 3). Tricuspid E/e' ratio and PAP gradually increased from controls, throughout women with gestational hypertension, to those with preeclampsia.

Global and free wall RV longitudinal strains, as well as corresponding endo- and epicardial strains, gradually decreased from controls, across patients with gestational hypertension, to women with preeclampsia (Table 3).

\section{Univariable and multivariable regression analysis}

LVEF, LVMI, PAP, 24-h systolic BP and visit-to-visit systolic BP and corresponding indices of $\mathrm{BP}$ variability were related with $\mathrm{RV}$ global and free wall longitudinal strains (Table 4). LVMI, 24-h systolic BP and both parameters of BP variability were associated with global and free wall RV longitudinal strains independently of other echocardiographic and demographic parameters (Models 1 and 2). Visit-to-visit systolic BP, as well as parameters of BP variability, was significantly and independently associated with global and free wall RV longitudinal strains (Models 3 and 4).

\section{DISCUSSION}

Our investigation revealed several important findings summarized as follows: (i) short- and long-term BP variability gradually and significantly increased from controls, across women with preeclampsia, to those with gestational hypertension; (ii) RV structural, functional and mechanical remodeling was found in patients with gestational hypertension and preeclampsia; (iii) parameters of short- and long-term BP variability were independently of demographic, clinical and echocardiographic parameters associated with RV global and free wall longitudinal strains. 
Table 4. Associations of $24-\mathrm{h}$ and visit-to-visit blood pressure variability indices and RV longitudinal strain (univariate and multivariate regression analysis).

\begin{tabular}{|c|c|c|c|c|c|c|c|c|c|c|}
\hline & \multicolumn{5}{|c|}{ RV global longitudinal strain (\%) } & \multicolumn{5}{|c|}{ RV free wall longitudinal strain (\%) } \\
\hline & $\boldsymbol{\beta}$ & $\beta$ (M1) & $\beta(M 2)$ & $\beta($ (M3) & $\beta$ (M4) & $\boldsymbol{\beta}$ & $\beta$ (M1) & $\beta$ (M2) & $\beta($ (M3) & $\beta$ (M4) \\
\hline Age (years) & -0.13 & -0.08 & -0.11 & -0.10 & -0.06 & -0.16 & -0.14 & -0.10 & -0.13 & -0.09 \\
\hline LVEF (\%) & $0.24^{\dagger}$ & 0.14 & 0.12 & 0.13 & 0.12 & $0.25^{\dagger}$ & 0.12 & 0.17 & $0.22^{\dagger}$ & 0.16 \\
\hline LVMI $\left(g / \mathrm{m}^{2}\right)$ & $-0.30^{\ddagger}$ & $-0.25^{\dagger}$ & $-0.27^{\dagger}$ & $-0.23^{\dagger}$ & $-0.31^{\neq}$ & $-0.30^{\ddagger}$ & $-0.26^{\dagger}$ & $-0.25^{\dagger}$ & $-0.25^{\neq}$ & $-0.24^{\dagger}$ \\
\hline $\mathrm{E} / \mathrm{e}_{\mathrm{m}}^{\prime}$ ratio & $-0.20^{\dagger}$ & -0.12 & -0.15 & -0.11 & -0.12 & $-0.21^{\dagger}$ & -0.15 & -0.13 & -0.15 & -0.12 \\
\hline 24-h SBP (mmHg) & $-0.32^{\ddagger}$ & $-0.31^{\dagger}$ & $-0.26^{\dagger}$ & - & - & $-0.26^{\dagger}$ & -0.15 & -0.16 & - & - \\
\hline SD (24-h SBP) & $-0.37^{\ddagger}$ & $-0.32^{\ddagger}$ & - & - & - & $-0.31^{\ddagger}$ & $-0.26^{\dagger}$ & - & - & - \\
\hline CV (24-h SBP) & $-0.39^{\neq}$ & - & $-0.31^{\ddagger}$ & - & - & $-0.29^{\ddagger}$ & - & $-0.26^{\dagger}$ & - & - \\
\hline V-to-V SBP (mmHg) & $-0.40^{\ddagger}$ & - & - & $-0.34^{\neq}$ & $-0.31^{\ddagger}$ & $-0.38^{\ddagger}$ & - & - & $-0.22^{\dagger}$ & $-0.30^{\ddagger}$ \\
\hline V-to-V SD (SBP) & $-0.35^{\ddagger}$ & - & - & $-0.27^{\ddagger}$ & - & $-0.30^{\ddagger}$ & - & - & $-0.29^{\dagger}$ & - \\
\hline
\end{tabular}

$C V$ coefficient of variation, $E$ early diastolic mitral flow (pulse Doppler), $e^{\prime}$ early diastolic flow velocity across the septal segment of mitral ( $e^{\prime}$ ) annulus (tissue Doppler), EF ejection fraction, LV left ventricle, LVMI left ventricular mass index, M1 model 1 (age, BMI, use of antihypertensive medications, LVEF, LVMI, E/e', 24h SBP, and SD (24-h SBP)), M2 model 2 (age, BMI, use of antihypertensive medications, LVEF, LVMI, E/e', 24-h SBP, and CV (24-h SBP)), M3 model 3 (age, BMl, use of antihypertensive medications, LVEF, LVMI, E/e', V-to-V SBP, and V-to-V SD (24-h SBP)), M4 model 4 (age, BMI, use of antihypertensive medications, LVEF, LVMI, E/e', V-to-V SBP, V-to-V CV (24-h SBP), and V-to-V ARV (SBP)), RV right ventricle, SBP systolic blood pressure, SD standard deviation, $V$-to-V visit to visit. $+p<0.05$. ${ }^{\ddagger} \mathrm{p}<0.01$.

Our findings showed that indices of short- and long-term of BP variability gradually increased from controls, across women with preeclampsia, to those with gestational hypertension. Large study that included almost 1000 patients with gestational hypertension and preeclampsia investigated visit-to-visit BP variability in second and third trimester and reported similar results with significantly higher BP variability among women with gestational hypertension and preeclampsia than in normotensive controls [12]. In maximally adjusted models, authors showed that visit-to-visit BP variability was associated with gestational hypertension and preeclampsia [12]. The novelty of our study is inclusion of short-term BP variability and conclusion that short- and long-term BP variability assessments could be interchangeably used in clinical practice for all women with hypertensive disorders in pregnancy.

Existing data show a significant discrepancy between studies regarding RV remodeling. Caglar et al. reported a significant enlargement of RV and RA, and deterioration of RV systolic and diastolic function in women with preeclampsia comparing with controls [19]. Some researches reported enlarged RV, increased pulmonary arterial pressure and reduced RV longitudinal strain in patients with pregnancy-induced hypertension [6-9], whereas others did not find significant difference between these patients and normotensive controls [10, 11].

Our results revealed that RV global and free wall longitudinal strains gradually decreased from controls, across women with gestational hypertension, to those with preeclampsia. The same results were obtained for RV endocardial and epicardial strains for the global and free wall RV. In patients with acute preeclampsia RV global strain was significantly lower than in normotensive controls and this was particularly noticed for basal and apical RV segments $[6,9]$. Yu et al. reported lower RV longitudinal strain in women with preeclampsia than in normotensive controls, [3], whereas other studies found no difference in RV global longitudinal strain $[8,11]$.

The novel finding of the present study is the evaluation of global and free wall RV longitudinal strain, as well as assessment of layer-specific strain in this population. This analysis enabled the detection of endo- and epicardial RV changes in patients with pregnancy-induced hypertension and revealed that all myocardial layers are equally affected in these patients. Moreover, 24-h and visit-to-visit systolic BP and BP variability were independently associated with RV global and free wall longitudinal strains.

RV longitudinal strain is still in normal range in our study population, but this is expected for hypertensive patients [20, 21]. However, the recent meta-analysis in COVID-19 patients revealed that each $1 \%$ decrease in RV longitudinal strain was associated with $25 \%$ higher risk of poor outcome (OR 1.25, 95\% Cl: 1.15-1.35, $p<0.001$ ) [22], which could have a great implication in our study that found $\sim 2 \%$ absolute reduction in RV longitudinal strain in women with gestational hypertension and almost $4 \%$ in patients with preeclampsia.

There are several potential mechanisms that could explain RV remodeling in our study population. RV enlargement and increased RV filling pressure (elevated E/e $e^{\prime}$ ) could be explained by the volumeoverloaded state in hypertensive pregnant women [23]. Increased pulmonary resistance in gestational hypertension and preeclampsia, the consequence of reduced LV compliance and increased LV diastolic filling pressures, might also induce the reduction in RV longitudinal strain [24]. This hypothesis was partially supported by increased mean pulmonary artery pressure in gestational hypertension and preeclampsia in our study. Previous study also reported that RV dysfunction was associated with elevated pulmonary vascular resistance due to increased LV filling pressure, which was also confirmed in our study [24]. Cardiac magnetic resonance detected LV interstitial edema in $40 \%$ of preeclamptic women and it is reasonable to hypothesize that similar changes occur in the RV, which might be responsible for deterioration of RV strain in women with gestational hypertension and preeclampsia.

Our findings have several important clinical implications. Shortterm BP variability, obtained from 24-h ABPM, is equally important as long-term BP variability derived from visit-to-visit BP measurements in prediction of RV remodeling in women with pregnancyinduced hypertension. This indicates that 24-h ABPM can be a reliable method for evaluation of BP and BP variability in pregnant women. This method is fast, inexpensive and widely available. Considering the relationship between parameters of BP variability and RV remodeling, our results indicate that impaired BP variability could predict the existence of subclinical impairment of RV mechanics, which might be partly responsible for cardiac 
complications, including heart failure during and after pregnancy. The reduction in RV longitudinal global and layer-specific strain that we found in pregnancy-induced hypertension is not the part of physiological adaptation to pregnancy and it might be associated with adverse outcome of patients. Nevertheless, follow-up data are missing and this should serve as hypothesis for further investigations in this field.

\section{LIMITATIONS}

The present study has several limitations. Visit-to-visit BP variability in pregnancy could be challenging due to difficulty to define a baseline BP from which to make comparisons. However, at least 2 BP measurements from the first 20 weeks of gestation were available and therefore this problem was overcome. Preeclampsia was diagnosed in majority of patients before 32 gestational weeks and therefore we could not compare RV remodeling between women with early and late preeclampsia. Data about altered uterine artery Doppler are not available and it was not possible to determine the cause responsible for preeclampsia: placental, maternal or both. The appropriate visualization of the RV for assessment of longitudinal strain in pregnant women, particularly in the last trimester, could be difficult, but we succeeded to make adequate acquisitions in almost all participants. Due to COVID-19 pandemic the follow-up of our patients was not performed and outcome is unknown, which is additional limitation of the present study. The causal relationship between BP variability and RV structure and function could not be adequately evaluated due to a cross-sectional nature of this investigation.

\section{CONCLUSION}

Women with gestational hypertension and preeclampsia had significantly higher short- and long-term BP variability than normotensive controls. RV global and free wall longitudinal strains gradually and significantly reduced from control subjects, throughout patients with gestational hypertension, to preeclamptic women. Endo- and epicardial RV longitudinal strains were equally affected in women with pregnancy-induced hypertension. Parameters of short- and long-term BP variability were independently of clinical and echocardiographic parameters and BP associated with global and free wall RV longitudinal strains. Our findings highlighted the importance of short- and long-term BP variability, which can be used interchangeably, as a significant set of parameters that may indicate pregnant women who are at risk of adverse RV remodeling during and after pregnancy. Longitudinal studies with larger number of patients are necessary to investigate the potential predictive role of BP variability and subclinical RV changes in women with pregnancy-induced hypertension on long-term outcomes.

\section{Summary table}

What is known about topic

- Pregnancy-induced hypertensive disorders are associated with adverse outcome

- Left ventricular mechanics may be impaired in pregnancyinduced hypertensive disorders

- Visit-to-visit blood pressure variability is increased in women with gestational hypertension and preeclampsia

What this study adds

- Right ventricular mechanics is deteriorated in women with gestational hypertension and preeclampsia in comparison to normotensive pregnant women
- Short-term blood pressure variability measured during 24-h blood pressure monitoring is also increased in women with gestational hypertension and preeclampsia

- Long- and short-term blood pressure variability parameters were associated with right ventricular mechanics in women with gestational hypertension and preeclampsia

\section{REFERENCES}

1. Lo JO, Mission JF, Caughey AB. Hypertensive disease of pregnancy and maternal mortality. Curr Opin Obstet Gynecol. 2013;25:124-32.

2. Theilen LH, Fraser A, Hollingshaus MS, Schliep KC, Varner MW, Smith KR, et al. Allcause and cause-specific mortality after hypertensive disease of pregnancy. Obstet Gynecol. 2016;128:238-44.

3. Yu L, Zhou Q, Peng Q, Zeng S, Yang Z. Velocity vector imaging echocardiography and NT-proBNP study of fetal cardiac function in pregnancy-induced maternal hypertension. J Clin Ultrasound. 2019;47:285-91.

4. Orabona R, Vizzardi E, Sciatti E, Bonadei I, Valcamonico A, Metra M, et al. Insights into cardiac alterations after pre-eclampsia: an echocardiographic study. Ultrasound Obstet Gynecol. 2017;49:124-33.

5. Liu W, Li Y, Wang W, Li J, Cong J. Layer-specific longitudinal strain analysis by speckle tracking echocardiography in women with early and late onset preeclampsia. Pregnancy Hypertens. 2019;17:172-177.

6. Vaught AJ, Kovell LC, Szymanski LM, Mayer SA, Seifert SM, Vaidya D, et al. Acute cardiac effects of severe pre-eclampsia. J Am Coll Cardiol. 2018; 72:1-11.

7. Mostafavi A, Tase Zar Y, Nikdoust F, Tabatabaei SA. Comparison of left ventricular systolic function by $2 \mathrm{D}$ speckle-tracking echocardiography between normal pregnant women and pregnant women with preeclampsia. J Cardiovasc Thorac Res. 2019;11:309-13.

8. Orabona R, Mohseni Z, Sciatti E, Mulder EG, Prefumo F, Lorusso R, et al. Maternal myocardial dysfunction after normotensive fetal growth restriction compared with hypertensive pregnancies: a speckle-tracking study. J Hypertens. 2020;38:1955-63.

9. Paudel A, Tigen K, Yoldemir T, Guclu M, Yildiz I, Cincin A, et al. The evaluation of ventricular functions by speckle tracking echocardiography in preeclamptic patients. Int J Cardiovasc Imaging. 2020;36:1689-94.

10. Chen SSM, Leeton L, Castro JM, Dennis AT. Myocardial tissue characterisation and detection of myocardial oedema by cardiovascular magnetic resonance in women with pre-eclampsia: a pilot study. Int J Obstet Anesth. 2018;36:56-65.

11. Breatnach CR, Monteith C, McSweeney L, Tully EC, Malone FD, Kent E, et al. The impact of maternal gestational hypertension and the use of antihypertensives on neonatal myocardial performance. Neonatology. 2018;113:21-26.

12. Jieyu L, Yingying $C$, Tian $G$, Jiaxiang $W$, Jiawen $L$, Yingjie $G$, et al. Visit-to-visit blood pressure variability is associated with gestational hypertension and preeclampsia. Pregnancy Hypertens. 2019;18:126-31.

13. Liu J, Yang L, Teng H, Cao Y, Wang J, Han B, et al. Visit-to-visit blood pressure variability and risk of adverse birth outcomes in pregnancies in East China. Hypertens Res. 2021;44:239-49.

14. Abdel Wahab MA, Farrag HM, Saied CE. 24-Hour blood pressure variability as a predictor of short-term echocardiographic changes in normotensive women with past history of preeclampsia/eclampsia. Pregnancy Hypertens. 2018;13:72-78.

15. National Institute for Health and Care Excellence. Hypertension in pregnancy: diagnosis and management (NICE guideline NG133). 2019. https://www.nice.org. uk/guidance/ng133.

16. Williams B, Mancia G, Spiering W, Agabiti Rosei E, Azizi M, Burnier M, et al. 2018 ESC/ESH Guidelines for the management of arterial hypertension. The Task Force for the management of arterial hypertension of the European Society of Cardiology and the European Society of Hypertension. J Hypertens. 2018;2018:1953-2041.

17. Lang RM, Badano LP, Mor-Avi V, Afilalo J, Armstrong A, Ernande L, et al. Recommendations for cardiac chamber quantification by echocardiography in adults: an update from the American society of echocardiography and the European association of cardiovascular imaging. J Am Soc Echocardiogr. 2015:28:1-39.

18. Rudski LG, Lai WW, Afilalo J, Hua L, Handschumacher MD, Chandrasekaran K, et al. Guidelines for the echocardiographic assessment of the right heart in adults: a report from the American Society of Echocardiography endorsed by the European Association of Echocardiography, a registered branch of the European 
Society of Cardiology, and the Canadian Society of Echocardiography. J Am Soc Echocardiogr. 2010;23:685-713.

19. Çağlar FN, Ozde C, Bostancı E, Çağlar IM, Çiftçi S, Unğan İ, et al. Assessment of right heart function in preeclampsia by echocardiography. Pregnancy Hypertens. 2016;6:89-94.

20. Tadic M, Cuspidi C, Pencic B, Jozika L, Celic V. Relationship between right ventricular remodeling and heart rate variability in arterial hypertension. J Hypertens. 2015;33:1090-7.

21. Tadic M, Cuspidi C, Pencic B, Sljivic A, Ivanovic B, Neskovic A, et al. High-normal blood pressure impacts the right heart mechanics: a three-dimensional echocardiography and two-dimensional speckle tracking imaging study. Blood Press Monit. 2014;19:145-52.

22. Wibowo A, Pranata R, Astuti A, Tiksnadi BB, Martanto E, Martha JW, et al. Left and right ventricular longitudinal strains are associated with poor outcome in COVID-19: a systematic review and meta-analysis. J Intensive Care. 2021;9:9.

23. Cotton DB, Lee W, Huhta JC, Dorman KF. Hemodynamic profile of severe pregnancy-induced hypertension. Am J Obstet Gynecol. 1988;158:523-9.
24. Melchiorre K, Sutherland GR, Watt-Coote I, Liberati M, Thilaganathan B. Severe myocardial impairment and chamber dysfunction in preterm preeclampsia. Hypertens Pregnancy. 2012;31:454-71.

\section{COMPETING INTERESTS}

MT is supported by the unrestricted Servier Research Grant. Other authors have not declared any conflicts of interest related to this study.

\section{ADDITIONAL INFORMATION}

Correspondence and requests for materials should be addressed to M.T.

Reprints and permission information is available at http://www.nature.com/reprints

Publisher's note Springer Nature remains neutral with regard to jurisdictional claims in published maps and institutional affiliations. 